\title{
Stellar Populations of the Most Massive Galaxies
}

\section{Alexander Fritz, Michael D. Hoenig, and Ricardo P. Schiavon}

Gemini Observatory, 670 N. A'ohoku Place, Hilo, HI 96720, USA

Email: afritz@gemini.edu

Keywords. galaxies: evolution, galaxies: structure - galaxies: elliptical and lenticular, cD

Within the hierarchical CDM framework, gas-poor mergers contribute substantially to the building of the most massive galaxies (Faber et al. 2007). We want to test this scenario by studying the fundamental plane (FP) and the stellar populations of the most massive galaxies. We investigate a well-defined sample of massive early-type galaxies at $0.1<z<0.4$, identified from the SDSS database. Out of 42,000 possible targets in the SDSS database, we extracted 23 luminous early-type galaxies with bona fide high velocity dispersions of $\sigma>350 \mathrm{~km} \mathrm{~s}^{-1}$. These systems are located either in high or low-density environments and show a variety of small surface-brightness structure. Using archival HST/ACS images and Gemini/GMOS spectroscopy, we will explore the photometric and spectroscopic properties of these galaxies.

These massive galaxies define steeper size, mass, and mass density-luminosity relations than the bulk of the SDSS early-type galaxy population (Bernardi et al. 2008). These results are consistent with dry mergers contributing importantly to the mass budget of massive galaxies. Figure 1 shows that the FP for our massive galaxies is tilted with respect to SDSS early-type galaxies. We will perform a stellar population analysis of high $S / N$ GMOS spectra to test whether this tilt results either from non-homology, variations in the dark matter content, or $M / L$ ratio among the galaxies.

AF acknowledges support from grant HST-GO-10826.01 from STScI.

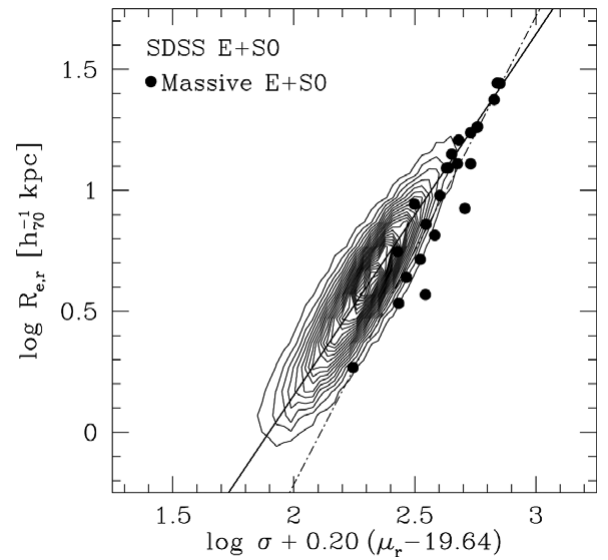

Figure 1. Edge-on view of the FP of most massive galaxies (circles) based on archival HST/ACS data and $\sigma$ from SDSS, compared to SDSS early-type galaxies (contours). The most massive galaxies follow a tilted FP independent from the choice of structural parameters ( $r^{1 / 4}$ or Sérsic).

\section{References}

Bernardi, M., et al. 2008, MNRAS, 391, 1191

Faber, S., et al. 2007, ApJ, 665, 265 